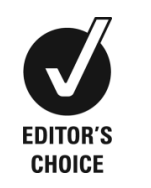

'Department of Oral Pathology and Microbiology, MES Dental College, Perinthalmanna, Kerala, India

${ }^{2}$ Oral and Maxillofacial Surgery, MES Dental College, Perinthalmanna, Kerala, India ${ }^{3}$ Department of Pedodontics, MES Dental College, Perinthalmanna, Kerala, India

Correspondence to Professor Abdul Hafiz hafizzzdr@gmail.com

Accepted 22 February 2016

\title{
Clinical diagnostic dilemma in an uncharacteristic rapidly enlarging swelling of the anterior maxilla: extranodal diffuse large B cell lymphoma
}

\author{
Cheriyanthal Sisupalan Jayapalan, ${ }^{1}$ Meera Kunjumon Pynadath, ${ }^{1}$ Ummar Mangalath, ${ }^{2}$ \\ Antony George, ${ }^{1}$ Sachin Aslam, ${ }^{1}$ Abdul Hafiz ${ }^{3}$
}

\section{SUMMARY}

Extranodal non-Hodgkin lymphoma (NHL) is infrequent in the oral cavity and constitutes $3.5 \%$ of oral cancers, and less than $2.2 \%$ of maxillofacial lymphomas. Diffuse large B cell lymphoma (DLBCL) accounts for $40 \%$ of $\mathrm{NHL}$ and has a 5 -year survival rate of less than $30 \%$. Early detection of extranodal NHL by dental personnel is extremely important as a delay in diagnosis can result in the cancer being diagnosed at an advanced stage and a poor prognosis. A 60-year-old male presented with an uncharacteristic asymptomatic rapidly enlarging swelling of the anterior maxilla, which on histopathological and immunohistochemical analysis was diagnosed as DLBCL. Imaging studies showed bone invasion and lymph node metastasis with poor prognosis. The patient received radiotherapy and chemotherapy but died within 3 months of diagnosis. A literature search revealed one another case with anterior maxilla occurrence, as the few oral DLBCL so far reported have appeared on the posterior palate or other intraoral sites.

\section{BACKGROUND}

Lymphomas are heterogeneous malignancies of the immune system that are classically grouped into Hodgkin lymphoma (HL) and non-Hodgkin lymphoma (NHL) based on differences in genetics, biology, histopathology, immunophenotype and clinical behaviour. ${ }^{1} \mathrm{HL}$ and NHL constitute 10 $14 \%$ and $86-90 \%$, respectively, of all lymphomas. ${ }^{12} \mathrm{HL}$ most commonly occurs in the cervical and mediastinal lymph nodes, whereas NHL commonly occurs in extranodal locations, with $2.2 \%$ appearing in the oral mucosa, jaw bones, salivary glands in the maxillofacial region, paranasal sinus, Waldeyer's ring, and the orbit. ${ }^{1}{ }^{2}$ Some $85-90 \%$ of all NHL arise from B lymphocytes and the rest from $\mathrm{T}$ cells and natural killer cells. ${ }^{2}$ Extranodal NHL is rare in the oral cavity, with the most frequently reported subtype being diffuse large B cell lymphoma (DLBCL). ${ }^{1} 2$ The most common sites are the palatal mucosa, gingiva, tongue, buccal mucosa, and floor of the mouth. ${ }^{1}$ DLBCL is most commonly seen in men above the age of 50 (6070) years. $^{2}$ The aetiology of DLBCL is uncertain and includes exposure to pesticides and radiation; long-term immunosuppression; autoimmune diseases such as rheumatoid arthritis, systemic lupus erythematosus and Sjögren syndrome; viruses including Epstein-Barr virus (EBV), human T cell lymphotropic virus (HTLV-1), HIV, human herpes virus (HHV-8), and hepatitis $\mathrm{B}$ and $\mathrm{C}$; and microorganisms like Helicobacter pylori and chlamydia. ${ }^{13}$ Typical oral symptoms include non-specific swelling, abscess, ulcer or a non-healing extraction socket. ${ }^{2}$ A literature review revealed that in the last 45 years fewer than 450 cases of primary oral NHL have been reported in the indexed scientific literature, although recently the incidence of lymphomas has been rapidly increasing to epidemic proportions worldwide. We describe an uncharacteristic, asymptomatic rapidly enlarging swelling of sudden onset on the anterior edentulous maxilla that resulted in a clinical diagnostic dilemma, and which on histopathological and immunohistochemical examination was reported as extranodal DLBCL.

\section{CASE PRESENTATION}

An unemployed semi-skilled man aged 60 years presented to our teaching institute with pain and a large swelling of the edentulous anterior maxilla. The swelling was sudden in onset and had increased rapidly in size (figure 1) in the previous 2 weeks. The patient gave a personal history of smoking 2-3 packets of beedi a day for the last 20 years. He was hypertensive and was currently on standard anti-hypertensive medication. Intraoral examination revealed a large lobular sessile ulcerated swelling of approximately $7 \times 3 \mathrm{~cm}$ in size and extending from the right second premolar to the left canine, involving the anterior hard palate and the labial vestibule (figure 2). The patient was edentulous except for the presence of grade III mobile right and left canines, left premolars, and left first molar. He gave no history of dental prosthesis for replacement of the missing teeth. On inspection and palpation, the soft tissue mass was non-tender, ulcerated with areas of redness, firm, and without thrill or pulsation. Multiple bilateral submandibular (level II) lymph nodes were palpable, and were non-tender, firm and fixed to the adjacent tissues.

\section{INVESTIGATIONS}

An investigatory orthopentograph and lateral cephalogram (figure 3 ) revealed diffuse poorly circumscribed radiolucency of the anterior maxilla with poor periodontal status of all the maxillary teeth. Routine blood investigations showed all parameters were within normal limits. An incisional scalpel biopsy was taken under local anaesthesia and sent for histopathological examination (HPE). $\mathrm{H} \& \mathrm{E}$ stained sections showed a stratified squamous 


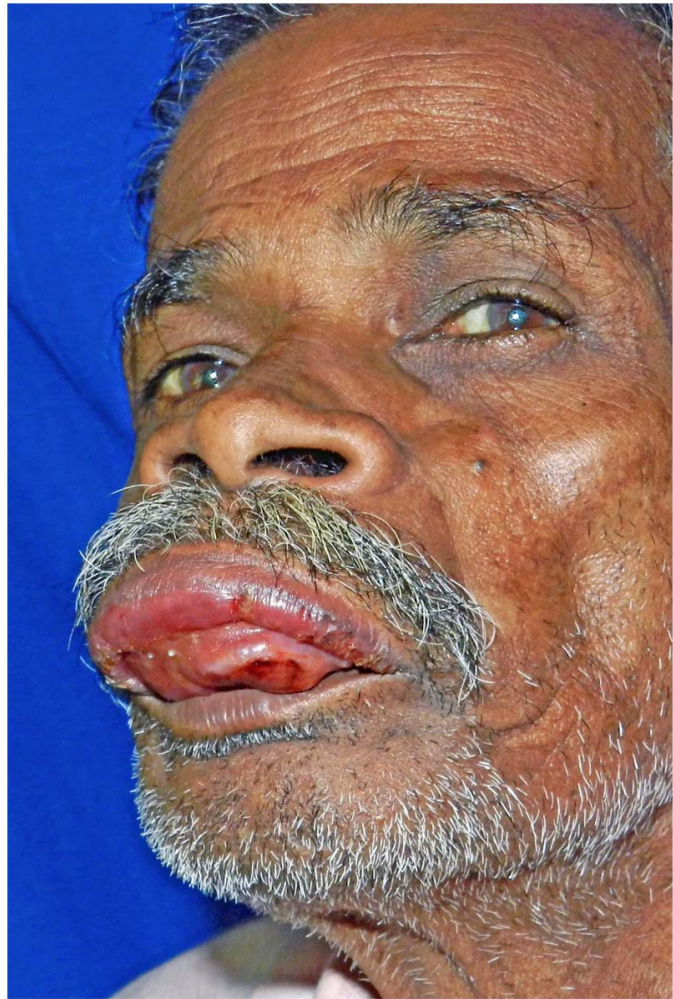

Figure 1 Extraoral view showing rapidly enlarging asymptomatic swelling of the anterior maxilla.

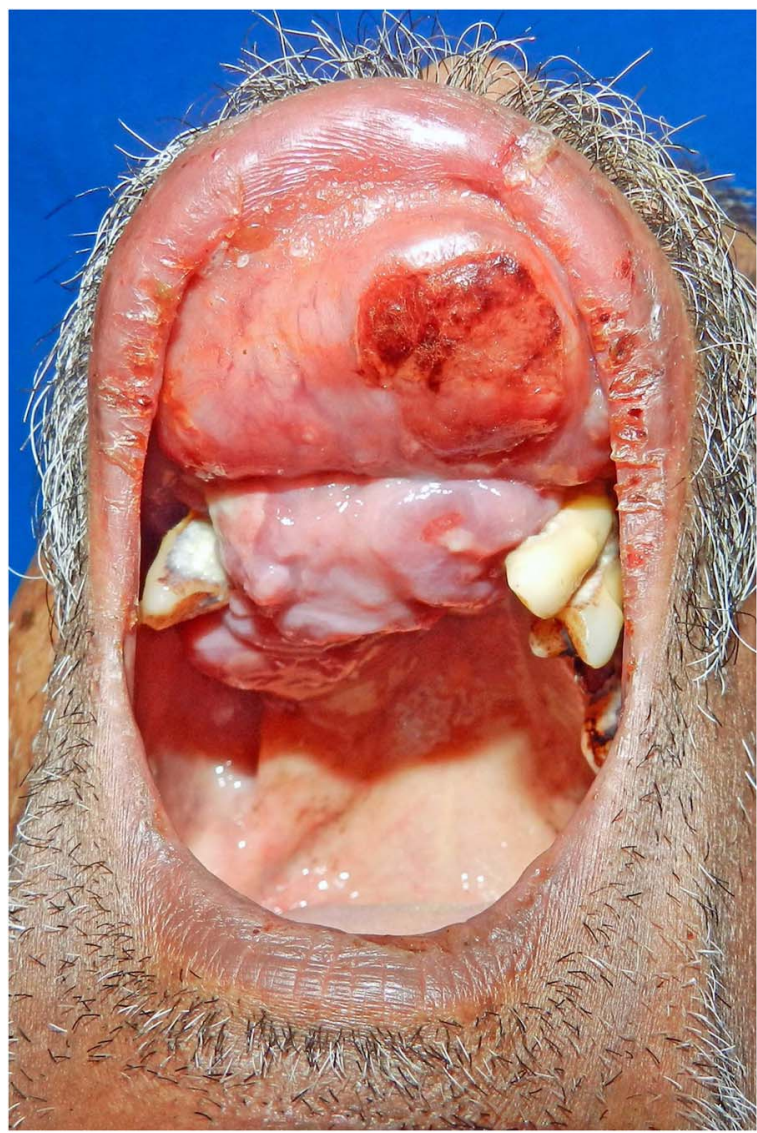

Figure 2 Intraoral view showing a lobular, ulcerated, firm swelling of the edentulous anterior hard palate and labial vestibule. surface epithelium with an underlying Grenz zone of fibrovascular connective tissue and deeper areas of dense hyperchromatic pleomorphic round cells with cleaved vesicular nuclei and prominent nucleoli (figures 4 and 5). A histopathological provisional diagnosis of round cell tumour, provisionally lymphoma, was made, and the tissue blocks were sent for immunohistochemistry (IHC). The tissues were negative for cytokeratin, desmin, S-100 and HMB-45, and showed 4+ (76-100\%) positive immunoreactivity to leucocyte antigen CD45 (figure 6). The second IHC panel showed negative immunoreactivity to CD3, Bcl-6 and CD138, and 4+ (76-100\%) positive immunoreactivity towards CD20 (figure 7). Based on the HPE and IHC findings, a histopathological diagnosis of extranodal DLBCL was made. The patient was advised to undergo a computerised tomography (CT scan) for further evaluation of the extent and spread of the lesion, but was not able to afford it, and so was referred to the government regional cancer centre (RCC).

\section{DIFFERENTIAL DIAGNOSIS}

Due to the clinical dilemma over the sudden uncharacteristic rapidly enlarging swelling of the anterior maxilla, a differential diagnosis of osteosarcoma, peripheral giant cell granuloma and squamous cell carcinoma was given by the examining oral and maxillofacial surgeon. After an incisional biopsy, a histopathological provisional diagnosis of lymphoma, to be confirmed by immunohistochemical markers, was made.

\section{TREATMENT}

The patient was advised to undergo chemotherapy and radiotherapy after evaluation of CT scans. However, due to his poor

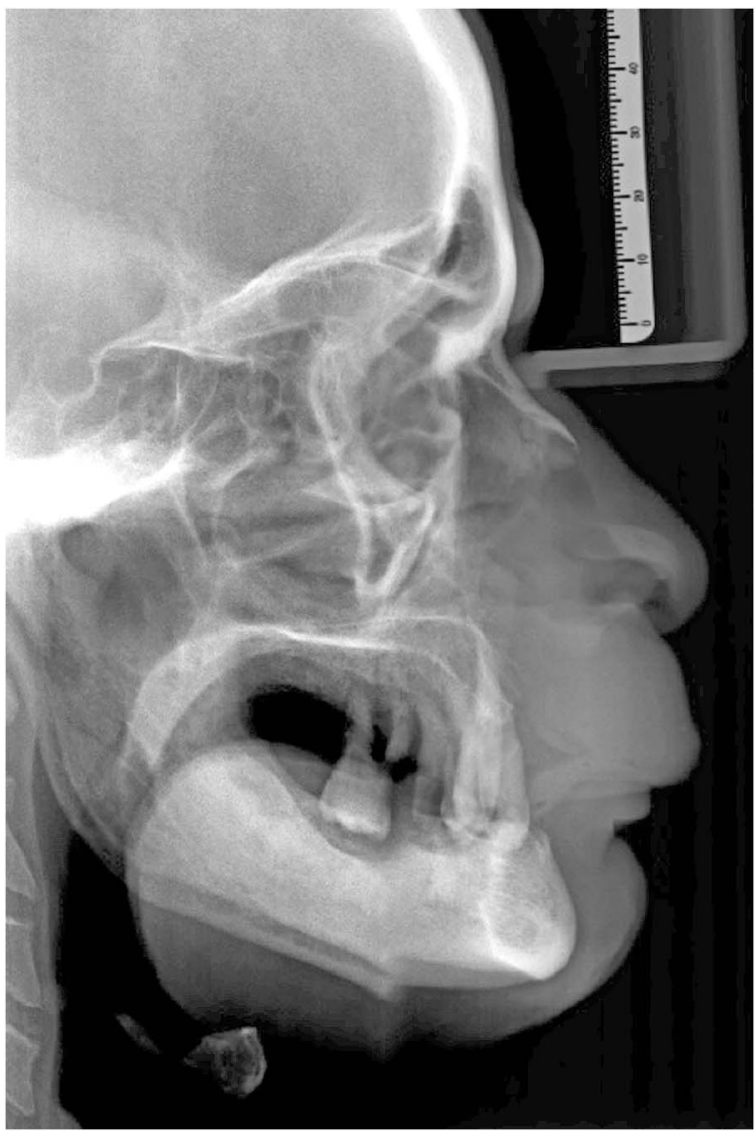

Figure 3 Lateral cephalogram showing the diffuse, poorly circumscribed radiolucency of the anterior maxilla with poor periodontal status of the remaining maxillary teeth. 


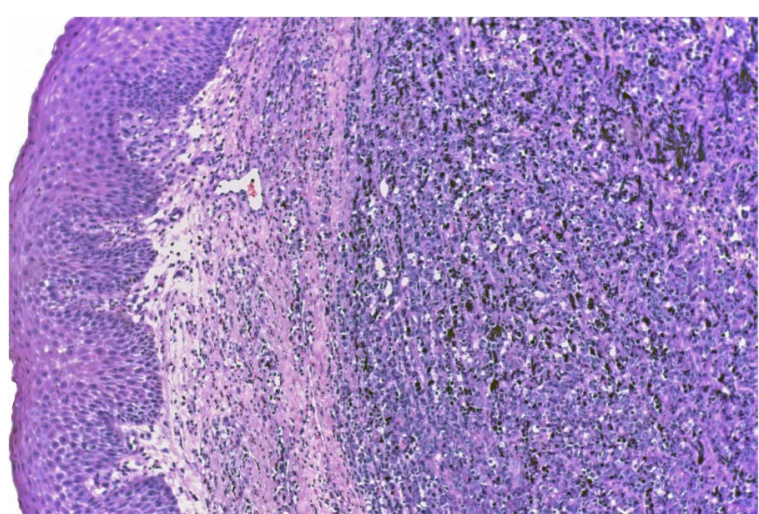

Figure 4 Photomicrograph $10 \times(\mathrm{H} \& \mathrm{E})$ of stratified squamous surface epithelium with underlying Grenz zone of fibrovascular connective tissue and deeper areas of lesional cells.

economic status and lack of medical insurance, he was unable to undergo treatment at our teaching hospital and so was referred to the RCC for treatment paid for by the government.

\section{OUTCOME AND FOLLOW-UP}

After presenting to the RCC the patient underwent further investigations including detailed imaging studies and our diagnosis of extranodal DLBCL was confirmed. He was declared to have a poor prognosis due to bone invasion, metastasis, and the aggressive behaviour of the lesion. He received chemotherapy and radiation (the detailed treatment plan is not known to the authors) at the RCC, but unfortunately died within 3 months of treatment initiation.

\section{DISCUSSION}

The incidence of NHL has been increasing globally since the 1970s, and is considered by some authors to be a worldwide epidemic, in contrast to the incidence of HL which is either decreasing or is stationary. ${ }^{3}$ DLBCL is heterogeneous regarding morphology, histopathology, immunophenotype, genetics and site of presentation. ${ }^{4}$ It is an aggressive malignant neoplasm of mature B lymphocytes and has been categorised based on cytomorphology into (1) centroblastic, (2) immunoblastic and (3) anaplastic types; and with the help of gene expression profiling (GEP) of the cell of origin into (1) activated B cell (ABC), (2) germinal centre $\mathrm{B}$ cell (GCB), and (3) type 3 gene expression

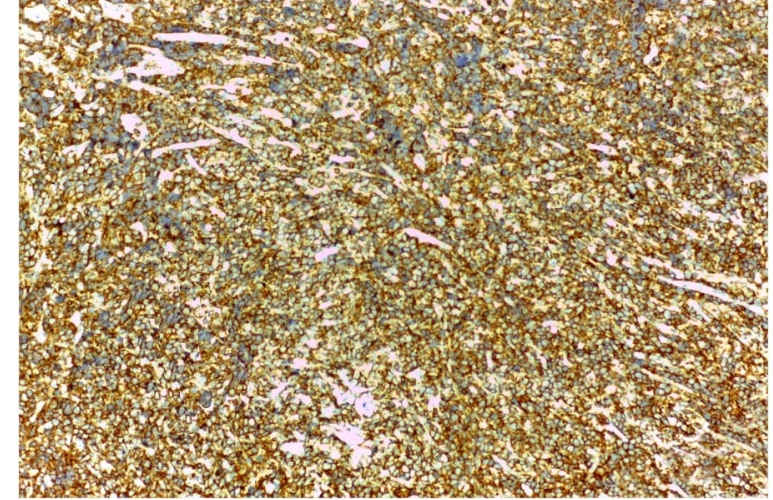

Figure 6 Photomicrograph 10x (immunohistochemistry) showing positive immunoreactivity to CD45.

profile groups. ${ }^{4} 5$ Even after the publication of the hierarchical classification by the Pathology Working Group of the International Lymphoma Epidemiology Consortium (InterLymph), the diagnosis and classification of lymphomas continue to be a challenge due to the large number of subtypes, their heterogeneous nature, and their complex relationships. DLBCL is slightly more common in men and commonly affects older adults in their sixth and seventh decades, although it can occur at any age and has been reported in children under 14 years of age. ${ }^{2}{ }^{5}$ In accordance with reports in the literature, our patient was a male aged 60 years. DLBCL of the head and neck may be associated with undiagnosed HIV, since they account for $2 \%$ of oral neoplasms in AIDS patients. ${ }^{6}$ The common presenting symptoms of oral NHL include a nontender swelling which grows rapidly, and may ulcerate and enlarge to become a necrotic and foul smelling lesion. ${ }^{3} 6$ Pain may develop if underlying bone is involved and is associated with tooth mobility and paraesthesia. ${ }^{3}$ Oral NHL is almost never accompanied by 'B' symptoms of fever, weight loss, night sweats, visceral pain and malaise. ${ }^{1}$ The most common site involved in the oral cavity is the posterior hard palate and buccal vestibule. $^{6} 7$ Our patient had no B symptoms and presented with a lobulated large swelling involving the anterior palate and labial vestibule with underlying bone erosion and metastasis to submandibular lymph nodes. A literature search revealed only one similar case of a lesion with anterior maxilla occurrence in a 76 -year-old male. ${ }^{8}$

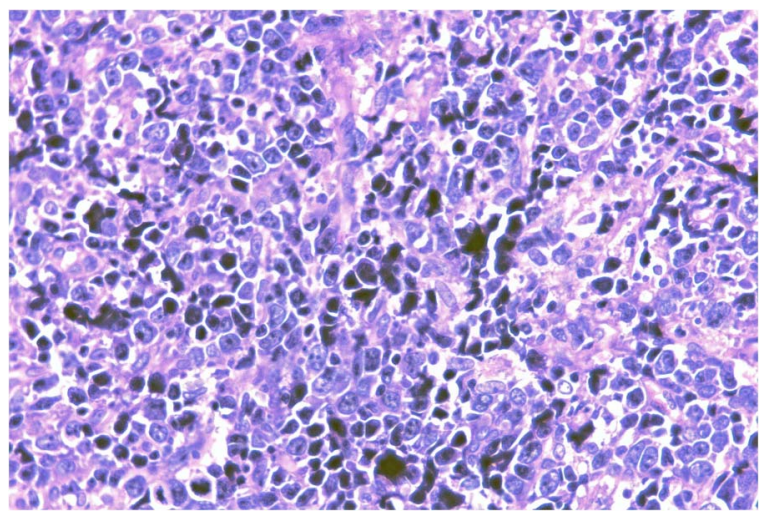

Figure 5 Photomicrograph 40× (H\&E) showing hyperchromatic pleomorphic round cells with cleaved vesicular nuclei and prominent nucleoli.

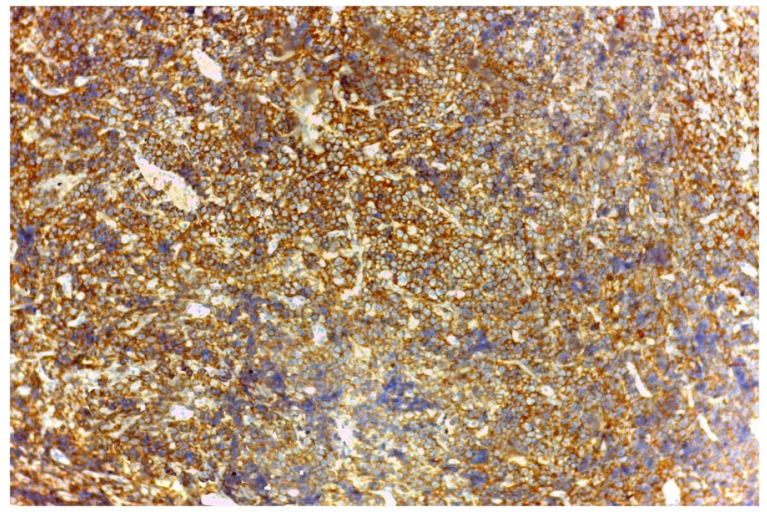

Figure 7 Photomicrograph 10x (immunohistochemistry) showing positive immunoreactivity to CD20. 
In non-immunocompromised individuals the most common lymphoma in the head and neck region is DLBCL, followed by mantle cell lymphoma, marginal zone B cell lymphoma and Burkitt's lymphoma. ${ }^{9}$ DLBCL characteristically expresses CD45 (leukocyte common antigen) and one or more pan-B cell markers: CD19, CD20, CD22, CD79 and PAX5. ${ }^{5} 6$ Other markers that have shown immunopositivity in DLBCL are CD10, Bcl6, Bcl2, Ki67, CD43, CD5, CD30, IRF4/MUM1 and p53. The presentation in our case is consistent with the reported literature and showed $76-100 \%$ immunopositivity for CD45 and CD20, and immunonegativity to CD3 (T cell marker), Bcl6 (GCB marker) and CD138 (plasma cell marker). CD20 is expressed on mature precursor B cells and is a highly specific marker for DLBCL. ${ }^{6}$ In immunoblastic, anaplastic or plasmablastic morphology, CD20 expression may be lost. ${ }^{6}$ Recently, researchers have concluded that the microenvironment and the inflammatory response may have a vital role in the prognosis of DLBCL, with cases positive for CD31 and VEGF having a poorer prognosis. ${ }^{5}$

There are few case reports of oral DLBCL in the scientific literature, which makes diagnosis, understanding of biological behaviour, prognosis and therapeutic options difficult. Current treatment for B cell lymphomas usually consists of three to six cycles of multi-agent chemotherapy with cyclophosphamide, hydroxydoxorubicin, oncovin, and prednisone (CHOP) or R-CHOP which also includes anti-CD20 rituximab. $^{1}{ }^{6-9}$ Early stage disease responds well to chemotherapy or a combination of chemotherapy and radiotherapy (28-40 Gy fractionated over 2-4 weeks) with a very limited role for surgery. ${ }^{6} 79$ Advanced stage disease or relapse usually requires further combinations with methotrexate, bleomycin, doxorubicin, vincristin, dexamethasone, ifosfamide, epirubicin, etoposide, and other chemosensitive therapeutic agents, and higher dose radiation. ${ }^{9}$ Only a few survival and prognostic factor studies have been carried out for oral NHL. The International Prognostic Index (IPI) is now widely used to provide relevant prognostic information, with age $>60$ years, Ann Arbor clinical stage of III or IV, more than one extranodal site of involvement, performance status $>2$, and elevated serum lactate dehydrogenase (LDH) showing poor prognosis. ${ }^{16} \mathrm{HIV}$ positive patients have $60 \%$ more risk for lymphomas and have a poorer prognosis. ${ }^{7}$ The 5 -year survival rate of patients with low and high IPI scores are $73 \%$ and $23 \%$, respectively. ${ }^{6}$ In comparison to Western populations, oral NHL in Indian subpopulations has been found to be more aggressive. ${ }^{7}$

\section{CONCLUSION}

The diagnosis of oral NHL and DLBCL is challenging for a dental surgeon in view of the low index of clinical suspicion due to its rarity, and the wide variation in clinical, radiographical and histopathological findings. Dental surgeons play an important role in the early diagnosis of oral NHL, and should have adequate knowledge of the clinical and pathological characteristics of DLBCL, as a delay in diagnosis can result in the cancer being recognised at an advanced stage with resultant poor prognosis. If there is a diagnostic dilemma over an uncharacteristic, asymptomatic, rapidly enlarging, non-specific oral swelling or ulcer of sudden onset, the dental surgeon should include DLBCL in their provisional diagnosis.

\section{Learning points}

- Although infrequent in this region, extranodal lymphoma should be included in the differential diagnosis for an uncharacteristic swelling of the anterior maxilla.

- Less than 450 cases of primary oral lymphomas have been reported in the scientific literature over the last 45 years, but the incidence of lymphoma has increased rapidly over the last decade.

- Dental surgeons play an important role in the early detection of extranodal non-Hodgkin's lymphoma of the head and neck region, and should be familiar with the clinical and pathological characteristics of these lesions.

- Early stage disease responds well to multi-agent chemotherapy with cyclophosphamide, hydroxydoxorubicin, oncovin and prednisone (CHOP) or CHOP with rituximab (R-CHOP) in combination with radiotherapy, but advanced stage disease or relapse has a poor prognosis.

Contributors CSJ, MKP and AG evaluated the case and made the histopathological diagnosis. UM and SA recorded the case history, performed clinical examinations, acquired patient consent, took photographs, decided on the clinical diagnosis, and made the incisional biopsy. MKP, AG and $A H$ reviewed and analysed the literature and collected material for the concept and drafting of the manuscript. All authors contributed actively to the conception, design, drafting and critical revision of the manuscript. All authors have read and approved the final version of the manuscript.

Competing interests None declared.

\section{Patient consent Obtained.}

Provenance and peer review Not commissioned; externally peer reviewed.

\section{REFERENCES}

1 Guevara-Canales JO, Morales-Vadillo R, Sacsaquispe-Contreras SJ, et al. Malignant lymphoma of the oral cavity and the maxillofacial region: overall survival prognostic factors. Med Oral Patol Oral Cir Bucal 2013;18:e619-26.

2 Walter C, Ziebart T, Sagheb K, et al. Malignant lymphomas in the head and neck region-a retrospective, single-center study over 41 years. Int J Med Sci 2015; 12:141-5.

3 Huh J. Epidemiologic overview of malignant lymphoma. Korean J Hematol 2012;47:92-104.

4 Cerhan JR, Kricker A, Paltiel O, et al. Medical history, lifestyle, family history, and occupational risk factors for diffuse large B-cell lymphoma: the interlymph non-Hodgkin lymphoma subtypes project. J Natl Cancer Inst Monogr 2014;48:15-25.

5 Said JW. Aggressive B-cell lymphomas: how many categories do we need? Mod Pathol 2013;26(Suppl 1):S42-56.

6 Bhattacharyya I, Chehal HK, Cohen DM, et al. Primary diffuse large B-Cell lymphoma of the oral cavity: germinal center classification. Head and Neck Pathol 2010;4:181-91.

7 Shah GH, Panwar SK, Chaturvedi PP, et al. Isolated primary extranodal lymphoma of the oral cavity: a series of 15 cases and review of literature from a tertiary care cancer centre in India. Indian J Med Paediatr Oncol 2011;32:76-81.

8 Sahoo SR, Misra SR, Mishra L, et al. Primary diffuse large B-cell lymphoma in the anterior hard palate: a rare case report with review of literature. J Oral Maxillofac Pathol 2014;18:102-6.

9 Sirsath NT, Lakshmaiah K C, Das U, et al. Primary extranodal non-Hodgkin's lymphoma of oral cavity A single centre retrospective study. J Can Res Ther 2014;10:945-50. 
Copyright 2016 BMJ Publishing Group. All rights reserved. For permission to reuse any of this content visit http://group.bmj.com/group/rights-licensing/permissions.

BMJ Case Report Fellows may re-use this article for personal use and teaching without any further permission.

Become a Fellow of BMJ Case Reports today and you can:

- Submit as many cases as you like

- Enjoy fast sympathetic peer review and rapid publication of accepted articles

- Access all the published articles

- Re-use any of the published material for personal use and teaching without further permission

For information on Institutional Fellowships contact consortiasales@bmjgroup.com

Visit casereports.bmj.com for more articles like this and to become a Fellow 